\section{Incidence Rates of Healthcare- Associated Infection in a Pediatric Home Healthcare Service}

\author{
André Ricardo Araujo da Silva, MD, $\mathrm{PhD}{ }^{1}$ \\ Cristina Vieira de Souza, RN; ${ }^{1}$ \\ Mario Eduardo Viana Guimarães, $\mathrm{MD}, \mathrm{PhD} ;^{1}$ \\ Guilherme Sargentelli, MD; \\ Marisa Zenaide Ribeiro Gomes, $\mathrm{MD}, \mathrm{MSc}, \mathrm{PhD}^{2, \mathrm{a}}$
}

We describe the incidence rates of home healthcare-associated infections (HHAIs) in a pediatric home healthcare service (PHHCS). The overall incidence density of HHAIs was 11.1 infections per 1,000 patient-days. Average incidence density of ventilator-associated pneumonia (VAP) was 6.8 per 1,000 ventilator-days. Strategies for control of VAP should be prioritized in PHHCSs.

Infect Control Hosp Epidemiol 2012;33(8):845-848

An estimated 1,459,900 patients received home healthcare assistance per day in the United States in 2007. ${ }^{1}$ Although estimates for the pediatric population are lower than for adults, the number of children in home healthcare services (HHCS) is increasing worldwide. ${ }^{2,3}$ The incidence density rates of home healthcare-associated infections (HHAIs) in children are not known, but some data are available. ${ }^{4,5}$ Araujo da Silva and colleagues previously reported that pneumonia is the principal cause of hospital readmission in children attended by a pediatric home healthcare service (PHHCS). ${ }^{4}$ In this article, we describe the incidence density of infections related to a PHHCS and their identified causative agents.

\section{METHODS}

We studied all patients (0-21 years) assisted for more than 24 hours between January 2008 and June 2009 by the PHHCS of Prontobaby Children's Hospital, a private pediatric hospital in Rio de Janeiro, Brazil. The healthcare assistance was performed at least 12 hours per day by a nurse and a weekly visit by a physician in the patient's home. Other healthcare specialists (physiotherapists or speech specialists) visited patients when necessary. All parents gave written consent for their children to participate in the study, which was approved by local institutional review board. The Infection Control Committee reviewed all PHHCS infection control protocols and gave periodic infection control training to physicians and nurses.

Surveillance of HHAI was performed by an infection control practitioner who performed weekly analysis of patient data collected daily by a nurse using an adaptation of the National Healthcare Safety Network's (NHSN) methodology. ${ }^{6}$
The adaptation concerned weekly, rather than daily, analysis of patients' medical records. The HHAI criteria were those recommended by APIC-HICPAC for home healthcare, ${ }^{7}$ with the inclusion of the following criteria for tachypnea: respiratory frequency higher than 50 breaths per minute for children aged 2-12 months and higher than 40 breaths per minute for children aged $1-5$ years.

Multidrug-resistant bacteria were identified according to published criteria. ${ }^{8}$ Routine screening for multidrug-resistant bacteria colonization was not performed in the PHHCS. Multidrug-resistant bacteria were investigated through clinical samples and rectal swabs when required by physicians in PHHCS or were systematically collected during readmissions to Prontobaby Hospital. ${ }^{4}$

Demographic variables were analyzed by descriptive statistics using EPI INFO (ver. 6.04; Centers for Disease Control and Prevention). Satterthwaite and $\chi^{2}$ tests were used to compare means and proportions. A 2 -sided $P$ value of .05 or less was considered statistically significant.

\section{RESULTS}

Thirty-one patients ( 16 males) were studied; average age at admission was 55 months (range, 4-231 months; median, 39 months). Cerebral palsy and bronchopulmonary dysplasia were principal causes of admission to PHHCS. ${ }^{4}$ Mechanical ventilation was required in 9 patients $(29 \%)$, whereas other invasive devices were never required.

The average incidence density of HHAI was 14.4 infections per 1,000 patient-days from September through December 2008 , whereas it was 10.6 infections $(P=.03)$ and 9.8 per 1,000 patient-days $(P=.009)$ during January through August 2008 and 2009, respectively (Figure 1). Respiratory infections were the main causes of infections (74/129 [57\%]), even in the period of higher HHAI rates (September-December 2008).

We observed 23 cases of ventilator-associated pneumonia (VAP), with 3,394 invasive ventilator-days, an average ventilator utilization ratio of $29 \%$ (range, $23 \%-39 \%$ ), and an average incidence density of 6.8 VAP cases per 1,000 ventilator-days. There was no statistically significant difference $(P=.66)$ in the incidence density of VAP between 2008 and 2009 (7.1 and 6 VAP cases/1,000 ventilator-days, respectively), although the average ventilator utilization ratios were $31 \%$ in 2008 and $26 \%$ in $2009(P=.08)$.

The etiologies of HHAI were identified in $11 \%(14 / 129)$ of infections (Table 1). None of the gram-negative bacteria had a multidrug-resistant profile, but $10 \%(3 / 31)$ of patients had multidrug-resistant bacterial colonization probably acquired during their PHHCS stays. ${ }^{4}$

Causes of death, which gives us the overall mortality rate 


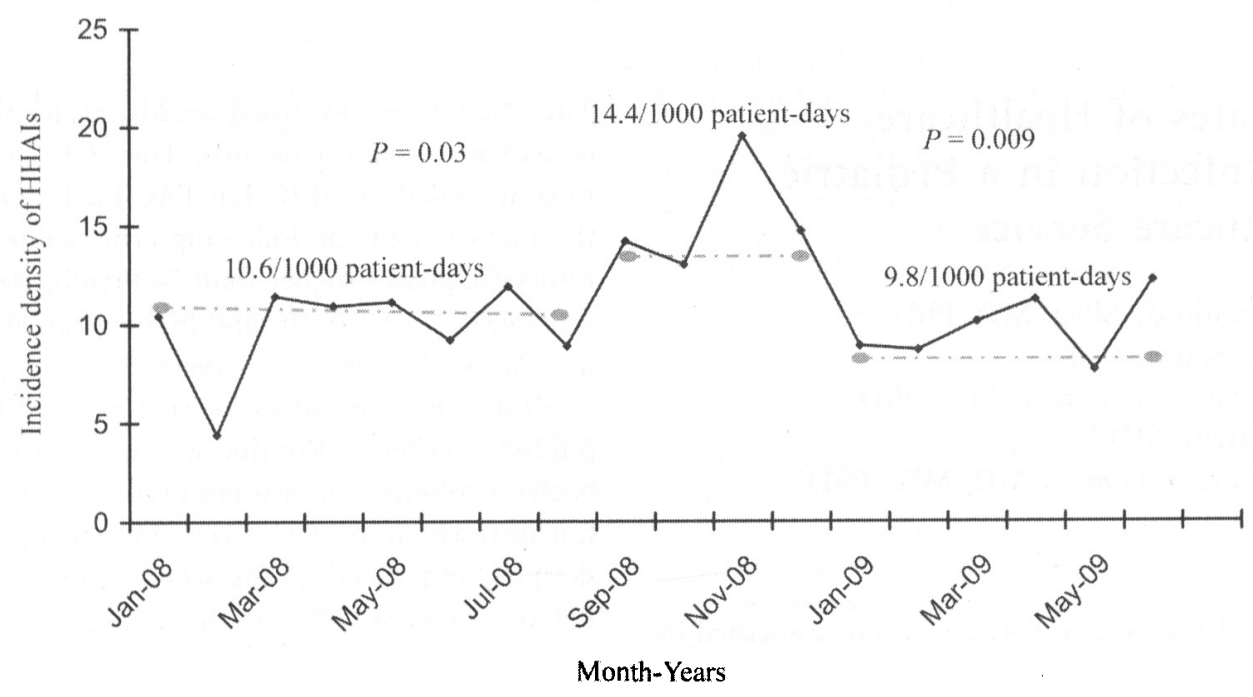

FIGURE 1. Incidence density (per 1,000 patient-days) of home healthcare-associated infections (HHAIs), pediatric home healthcare service of Prontobaby Children's Hospital, Rio de Janeiro, between January 2008 and June 2009. The solid lines show the average incidence rates of HHAIs for the periods. $P$ values for the difference among periods of higher and lower HHAI rates are shown.

of $9.7 \%$ and the HHAI-attributable mortality rate of $6.5 \%$, were previous described (Table 1$){ }^{4}$

\section{DISCUSSION}

Criteria and preventive measures for HHAI come from definitions or recommendations designed for hospitals, and these are frequently not useful for $\mathrm{HHCS}^{7}$ This is especially important in view of the unusual nature of this medical care, in which clinical staff fully interact and participate in the routine of family life, which does not occur in hospitals. Another poorly recognized aspect of home healthcare assistance is the risk of transference of microorganisms from hospital to the community through, for example, medical equipment or healthcare professionals serving as carriers. The patients themselves are probably the main reservoir of microorganisms, and despite their transference from hospital to home they are still dependent on invasive devices.

To the best of our knowledge, this is the first study that seeks to qualify and quantify HHAI in a PHHCS. Pneumonia and influenza-like illnesses accounted for more than $50 \%$ of all HHAIs, and VAP was the only cause of device-associated infections, because other devices were not required in our studied population. The anatomic changes of the thorax ( $\mathrm{sco}$ liosis, kyphosis, and kyphoscoliosis) and chronic bedridden conditions may have contributed to their acquisition of respiratory infections. VAP rates were higher than those tabulated by NHSN for pediatric critical units ${ }^{5}$ and for adults in HHCS, although the attributable mortality rates were similar. ${ }^{8}$ This result is in itself surprising, indicating a high risk for VAP in PHHCS. Despite using a different population and methodology for surveillance and diagnosis of VAP, the comparison with the NHSN rates, although not adequate, may serve as initial reference to emphasize the importance of establishing measures for VAP prevention in PHHCSs.

HHCSs have been considered cost-effective alternatives for patients with chronic diseases and those dependent on invasive devices in hospitals. ${ }^{9}$ However, analysis of overall incidence density of HHAI in our study showed rates of VAP comparable to nosocomial rates. Therefore, patients assisted in home healthcare require specific conduct standards: quality control of equipment and devices, cleanliness of the home, training in infection control for parents or caregivers, ${ }^{10}$ and standards for circulating healthcare professionals, who most often also work at hospitals. HHAI rates declining in 2009 was probably due to initial intervention after preliminary findings with hiring an administrative employee and standardization of procedures.

In this real-life study, the etiological agents were identified in only $11 \%$ of HHAIs, and gram-negative bacteria were the most identified cause of infections. The criteria for HHAI on the basis of clinical findings only, without complementary examinations, were insufficient for the diagnosis of infections. Microbiological and imaging examinations are difficult to perform in home settings, but they are nonetheless important and should be included. Additionally, although the infection criteria defined by the Association for Professionals in Infection Control and Epidemiology on the basis of clinical diagnoses of infections have practical advantages, it is also necessary to include other clinical parameters as we did for pneumonia. Another point is the difficulty to differentiate community infections from HHAI (all were considered as HHAI in our study). Moreover, besides the small sample size, HHAI surveillance was performed by the infection control practitioner weekly in patients' homes, although data were 
TABLE 1. Incidence Density and Etiology of HHAIs in 31 Patients, Pediatric Home Healthcare Service of Prontobaby Children's Hospital, between January 2008 and June 2009

\begin{tabular}{|c|c|c|c|}
\hline Types of infections & HHAIs & $\begin{array}{l}\text { Incidence density } \\
\text { of HHAI per } 1,000 \\
\text { patient-days }\end{array}$ & $\begin{array}{l}\text { Identified agents of HHAI } \\
\text { (no. of cases) }\end{array}$ \\
\hline Non-VAP & $32(24.8)^{b}$ & 2.7 & $\ldots$ \\
\hline VAP & $23(17.8)$ & $6.8^{\mathrm{c}}$ & $\begin{array}{l}\text { Pseudomonas sp. }(1)^{\mathrm{d}} \\
\text { Proteus sp. }(1)^{\mathrm{d}} \\
\text { Enterobacter } \mathrm{sp} .(1)^{\mathrm{d}}\end{array}$ \\
\hline Influenza-like illnesses & $19(14.7)$ & 1.6 & $\ldots$ \\
\hline Cutaneous infections & $18(14.0)$ & 1.6 & Candida spp. (2) \\
\hline Urinary tract infections & $15(11.6)^{\mathbf{b}}$ & 1.3 & $\begin{array}{l}\text { Klebsiella pneumonia (3) } \\
\text { Escherichia coli (2) } \\
\text { Morganella morgannii (2) }\end{array}$ \\
\hline Conjunctivitis & $9(7.0)$ & 0.8 & $\ldots$ \\
\hline Other infections & $13(10.1)$ & $1.1^{\mathrm{b}}$ & \\
\hline Bloodstream infections & 1 & 0.08 & Coagulase-negative staphylococci (1) \\
\hline Gastroenteritis & 4 & 0.3 & Rotavirus (1) \\
\hline Oral infections & 4 & 0.3 & $\ldots$ \\
\hline Otitis & 4 & 0.3 & $\ldots$ \\
\hline Total & $129(100)$ & 11.1 & $\ldots$ \\
\hline
\end{tabular}

Note. Data are no. (\%) of participants, unless otherwise noted. HHAI, home healthcare-associated infections; VAP, ventilator associated pneumonia.

a During readmissions to the hospital, patients were excluded for the calculation of number of patient-days.

b One case of death related to this type of infection.

c Per 1,000 ventilator-days.

d Tracheal aspirate.

collected daily by healthcare workers. This latter discrepancy may have contributed to the lack of the diagnoses for shortterm infections, and both conditions represent limitations of this study.

In conclusion, preventive measures that focus on healthcare-associated pneumonia are of great importance for children in HHCS. The use of written protocols and continuous training of the entire assisting team (including parents and caregivers) are recommended. Further studies should focus on dynamics of multidrug-resistant bacteria acquisition to better determine, for example, requirements for contact precautions for patients in PHHCS.

\section{ACKNOWLEDGMENTS}

Potential conflicts of interest. All authors report no conflicts of interest relevant to this article. All authors submitted the ICMJE Form for Disclosure of Potential Conflicts of Interest, and the conflicts that the editors consider relevant to this article are disclosed here.

Affiliations: 1. Hospital Infection Control Committee, Prontobaby Hospital da Criança, Rio de Janeiro, Brazil; 2. Nosocomial Infection Research Laboratory, Instituto Oswaldo Cruz, Fundação Oswaldo Cruz (FIOCRUZ), Rio de Janeiro, Brazil.

a Present affiliation: Marisa Zenaide Ribeiro Gomes, MD, MSc, PhD, Department of Infectious Disease, Infection Control and Employee Health, University of Texas MD Anderson Cancer Center, Houston, Texas.

Address correspondence to Marisa Zenaide Ribeiro Gomes, MD, MSc, $\mathrm{PhD}$, Nosocomial Infection Research Laboratory, Instituto Oswaldo Cruz,
Fundação Oswaldo Cruz, Avenida Brasil 4365, Pavilhão Rocha Lima S319, Manguinhos, Rio de Janeiro 21040-900, Brazil (marisargomes@hotmail.com, mrgomes@mdanderson.org).

Presented in part: 48th Annual Meeting of the Infectious Diseases Society of America; Vancouver, Canada; October 21-24, 2010.

Received December 6, 2011; accepted March 11, 2012; electronically published June 20, 2012.

(C) 2012 by The Society for Healthcare Epidemiology of America. All rights reserved. 0899-823X/2012/3308-0013\$15.00. DOI: 10.1086/666627

\section{REFERENCES}

1. Caffrey C, Sengupta M, Moss A, Harris-Kojetin L, Valverde R. Home Health Care and Discharged Hospice Care Patients: United States, 2000 and 2007. National Health Statistics Reports. Centers for Disease Control. April 2011; Number 38.

2. Wallis C, Paton JY, Beaton S, Jardine E. Children on long-term ventilatory support: 10 years of progress. Arch Dis Child 2011; 96:998-1002.

3. Hack M, Taylor HG, Drotar D, et al. Chronic conditions, functional limitations, and special health care needs of school-aged children born with extremely low-birth-weight in the 1990s. JAMA 2005;294:318-325.

4. Araujo da Silva AR, Vieira de Souza C, Viana ME, Sargentelli G, de Andrada Serpa MJ, Gomes MZR. Health care-associated infection and hospital readmission in a home care service for children. Am J Infect Control 2011;40:283-283.

5. Appierto L, Cori M, Bianchi R, et al. Home care for chronic respiratory failure in children: 15 years experience. Paediatr Anaesth 2002;12:345-350.

6. National Healthcare Safety Network (NHSN) Report. Data sum- 
mary for 2006 through 2007, issued November 2008. Am J Infect Control 2008;36:609-626.

7. APIC-HICPAC. Surveillance Definitions for Home Health Care and Home Hospice Infections. Association for Professionals in Infection Control and Epidemiology (APIC) and Healthcare Infection Control Practices Advisory Committee (HICPAC), February 2008, EUA 2008.

8. Siegel JD, Rhinehart E, Jackson M, Chiarello L. Management of multi drug-resistant organisms in healthcare settings, 2006. Am J Infect Control 2007;35(suppl 2):S165-S193.

9. Jones J, Wilson A, Parker $\mathrm{H}$, et al. Economic evaluation of hospital at home versus hospital care: cost minimisation analysis of data from randomised controlled trial. BMJ 1999;319: 1547-1550.

10. Edwards EA, O'Toole M, Wallis C. Sending children home on tracheostomy dependent ventilation: pitfalls and outcomes. Arch Dis Child 2004;89:251-255. 